\title{
Early life and socio-economic determinants of dietary trajectories in infancy and early childhood - results from the HSHK birth cohort study
}

Narendar Manohar ${ }^{1,2,3}$ (D) Andrew Hayen ${ }^{4}$, Loc Do ${ }^{5,6}$, Jane Scott ${ }^{7}$, Sameer Bhole ${ }^{8,9}$ and Amit Arora $1,2,8,10,11^{*}$

\begin{abstract}
Background: Early childhood is a period when dietary behaviours are established. This study aimed to examine the longitudinal intake of core and discretionary foods and identify early life and socio-economic factors influencing those intakes.

Methods: Mother-infant dyads ( $n=934)$ from the Healthy Smiles Healthy Kids study, an ongoing birth cohort study, were interviewed. The information on 'weekly frequency of core and discretionary foods intake' using a food frequency questionnaire was collected at 4 months, 8 months, 1 year, 2 years and 3 years age points. Group-based trajectory modelling analyses were performed to identify diet trajectories for 'core' and 'discretionary' foods respectively. A multinomial logistic regression was performed to identify the maternal and child-related predictors of resulting trajectories.

Results: The intake of core and discretionary foods each showed distinct quadratic $(n=3)$ trajectories with age. Overall, core foods intake increased rapidly in the first year of life, followed by a decline after age two, whereas discretionary foods intake increased steadily across the five age points. Multiparity (Relative Risk (RR): 0.46, 95\%Cl: 0.27-0.77), non-English speaking ethnicity of mother (RR: 0.66, 95\%Cl: $0.47-0.91$ ) and having a single mother (RR: 0.40, 95\% Cl: 0.18-0.85) were associated with low trajectories of core foods intake whereas older maternal age (RR: 1.05, 95\%Cl: 1.01-1.08) and longer breastfeeding duration (RR: 1.02, 95\%Cl: 1.00-1.03) were associated with higher trajectories of core foods intake. Also, multiparity (RR 2.63,95\%Cl: 1.47-4.70), low maternal education (RR 3.01, 95\%Cl: 1.61-5.65), and socio-economic disadvantage (RR 2.69, 95\%Cl: 1.31-5.55) were associated with high trajectories of discretionary foods intake. Conversely, longer duration of breastfeeding (RR 0.99, 95\%Cl: 0.97-0.99), and timely introduction of complementary foods (RR 0.30,95\%Cl: 0.15-0.61) had a protective effect against high discretionary foods consumption in infancy and early childhood.
\end{abstract}

\footnotetext{
* Correspondence: a.arora@westernsydney.edu.au

${ }^{1}$ School of Health Sciences, Western Sydney University, Penrith, NSW 2751,

Australia

${ }^{2}$ Health Equity Laboratory, Campbelltown, NSW 2560, Australia

Full list of author information is available at the end of the article
}

(C) The Author(s). 2021 Open Access This article is licensed under a Creative Commons Attribution 4.0 International License, which permits use, sharing, adaptation, distribution and reproduction in any medium or format, as long as you give appropriate credit to the original author(s) and the source, provide a link to the Creative Commons licence, and indicate if changes were made. The images or other third party material in this article are included in the article's Creative Commons. licence, unless indicated otherwise in a credit line to the material. If material is not included in the article's Creative Commons licence and your intended use is not permitted by statutory regulation or exceeds the permitted use, you will need to obtain permission directly from the copyright holder. To view a copy of this licence, visit http://creativecommons.org/licenses/by/4.0/. The Creative Commons Public Domain Dedication waiver (http://creativecommons.org/publicdomain/zero/1.0/) applies to the data made available in this article, unless otherwise stated in a credit line to the data. 
Conclusion: Children's frequency of discretionary foods intake increases markedly as they transition from infancy to preschool age, and the trajectories of intake established during early childhood are strongly influenced by sociodemographic factors and infant feeding choices. Hence, there is a need for targeted strategies to improve nutrition in early childhood and ultimately prevent the incidence of chronic diseases in children.

Keywords: Diet, Food frequency questionnaire, Trajectories, Patterns, Social determinants of health, Socio-economic inequality, Healthy lifestyle, Preschool children

\section{Background}

Infancy and early childhood is a period of rapid growth and development, coupled with evolving dietary requirements and physiological needs [1]. It is also a critical period during which susceptibility to many chronic diseases is established. An optimal diet in the early years is essential for a child's growth and development [2]. Additionally, dietary habits in early childhood lay the foundation for lifelong dietary preferences [3], and contribute to several health conditions such as obesity, dental caries, diabetes and metabolic syndrome $[4,5]$. Hence, adopting healthy dietary habits in early childhood and identifying population groups with sub-optimal dietary patterns early in life are important for preventing or at least delaying the incidence of chronic diseases [6]. Furthermore, understanding the complexity of dietary patterns and the factors influencing these patterns may help in defining which foods and/or diets are amenable to change and at what stage of life.

The transitional period from infancy to early childhood, accompanied by social and educational development is an important period for establishing dietary patterns that may continue into adulthood [7]. Furthermore, parental practices during this period can serve as a model of dietary behaviours for the next generation [8]. Much attention has been directed towards developing healthy diet practices in children, however more research needs to be undertaken to understand the long-term dietary patterns or dietary 'trajectories' in early childhood. The term 'trajectories' is defined as 'groups of individuals following similar patterns of a behaviour or outcome of interest over time' [9].

In recent years, dietary patterns in children have been examined using statistical approaches such as Principal Component Analysis (PCA), factor analysis and cluster analysis [10-14]. These techniques capture the whole diet in combination rather than individual food items and enable the identification of factors associated with dietary patterns of children. Studies using such approaches have shown that children from high socioeconomic status (SES) tend to consume higher quality diets compared to children from lower SES. Furthermore, maternal factors such as young age, lower education, unemployment, lower household income, multiparity, and smoking are predictors for unhealthy dietary patterns in children [10-14].
Lately, an innovative statistical approach known as Group-Based Trajectory Modelling (GBTM) has gained attention in health and clinical sciences research. The GBTM identifies clusters of individuals who follow similar trajectories of health behaviours over time [15]. A recent study from Longitudinal Study of Australian Children (LSAC) used GBTM to derive and compare longitudinal dietary patterns in two cohorts of children [16]. However, to the best of our knowledge, no study has examined GBTMderived dietary patterns of Australian children (birth3 years) and their determinants. The objectives of this study are to:

1. Describe the longitudinal dietary trajectories of core and discretionary foods of Australian children from birth to age 3 years; and

2. Identify the maternal and child-related determinants of the observed trajectories.

\section{Methods}

\section{Data and participants}

This study used prospective data collected from 2009/10 up to 2013, from the Healthy Smiles Healthy Kids (HSHK) birth-cohort study [17] in South Western Sydney (SWS). This cohort study has been well-described in earlier publications $[17,18]$. In summary, the study sample comprised of mothers who gave birth to live infants (with no known medical condition and no physical or intellectual disability which was likely to influence dietary behaviours, hygiene practices and physical activity), between October 2009 and February 2010, in public hospitals located within the Sydney and South Western Sydney Local Health Districts (formerly known as Sydney South West Area Health Service).

Mother-infant dyads $(n=1035)$ were recruited during their first post-natal visit (4 to 6 weeks postpartum) by Child and Family Health Nurses (CFHNs), who explained the study and obtained written consent for participation. For non-English speaking participants, interpreter services and written material in the native language of major ethnic groups (i.e., Vietnamese, Arabic, Hindi, Assyrian, Cambodian, Cantonese, Mandarin, Samoan) were provided. 


\section{Data collection}

At 8 weeks postpartum, the first (baseline) telephone interview was conducted to record information mainly on socio-demographic characteristics and infant-feeding practices including breastfeeding and use of formula at that age point. Subsequently, five follow-up telephone interviews were conducted at 4 months, 8 months, 1 year, 2 years, and 3 years age points. Considering early identification of infant feeding practices was one of the primary objectives of the HSHK birth cohort study, the first follow-up interview was undertaken at 4 months because this is a period of transition from breastfeeding to solid (complementary) foods and variations existed in the international and national infant feeding guidelines and practices of that time. For example, although the World Health Organization (WHO) [19] and Australian National Health and Medical Research Council (NHMR C) [20] recommended that infants should be exclusively breastfed for 6 months and that complementary foods be introduced thereafter. The majority of Australian infants (91.5\%) receive complementary foods prior to 6 months and just over one third (35.3\%) by 4 months of age [21]. The questionnaire used in this study was adapted from the Iowa Fluoride study [22], the NSW Child Health Questionnaire [23], the National Child Oral Health Survey [24], Perth Infant Feeding Studies (PIFS I and II) $[25,26]$, and the HSHK pilot study [27].

\section{Outcome measures}

\section{Dietary intake assessment}

At every follow-up interview, information on the child's current dietary habits in terms of consumption of 32 individual food and drink items during the preceding 7 days was obtained from the mother. At each interview, a short food frequency questionnaire (FFQ) was used (see Additional file 1) and mothers were asked an openended question "In the past 7 days how often was your baby/child fed each of the following foods and/or drinks?". A numerical response was recorded to represent the number of times the specified food and/or drink was eaten in a week.

The 32 listed foods (see Additional file 1) were categorised into 'core' and 'discretionary' foods based on the Australian Dietary Guidelines [28, 29]. The same method was used to categorise the foods in a previously published research [30]. Core foods ( $n=12$ food items) comprised of five food sub-groups: dairy (e.g., milk, cheese, plain yoghurt) ( $n=4$ food items), grains (e.g., cereals, bread, rice) ( $n=3$ food items), fruits, vegetables, and meat and its alternatives (e.g., red meat, poultry, fish, and eggs) ( $n=3$ food items). The discretionary foods ( $n=20$ food items) were categorised into two subgroups: foods high in saturated fats and/or salt (e.g., potato chips and savoury snacks) ( $n=2$ food items), and foods and drinks with added sugars (e.g., fruit juice, confectionary, biscuits, cakes, sugar-sweetened beverages (SSBs), sweetened yogurt, ice cream) ( $n=18$ food items). Fruit juices were considered discretionary (specifically sugar-based) because of their high total sugar and energy value [28].

Diet can be summarised in different ways, such as dietary patterns, indices, or scores [31]. Dietary scores sum the number or frequency of foods consumed during a specified time that are considered to be healthy or unhealthy [31]. These scores are intuitive and analytically simple therefore, were used to construct dietary trajectories. In this study, for dietary trajectory analyses across the five specified time-points, the seven mutually exclusive food groups were collapsed into two major food groups i.e., core ( $n=12$ food items) and discretionary ( $n=20$ food items). The frequency (continuous data) of each food in the five individual core food groups and two discretionary food groups were summed, and then the totals of each of the five individual core food groups were summed to give the 'frequency of total core food group intake' and then the total frequency of the two discretionary groups were summed to give the 'total of the discretionary food group frequency'. Hence, individual dietary patterns were developed for the two major food groups and the seven individual food sub-groups respectively. The focus of the present study was primarily on the two major food groups i.e., core and discretionary foods.

\section{Predictors}

Several maternal and child factors considered to be potential determinants of healthy and unhealthy dietary patterns in children were derived from the literature [10-14] and investigated in the analyses. Maternal factors were: mother's age at child's birth (in years), marital status (single or married/living with partner), level of education (<Year 12, completed school, college, or university), employment status at 4 months postpartum (not working or working), mother's country of birth (Australia, other English-speaking, or non-English-speaking), number of children in household $(1,2$, or $\geq 3)$, and area-level socioeconomic status (SES) (deciles 9-10= least disadvantaged, deciles 7-8= low disadvantaged, deciles 5-6= moderately disadvantaged, deciles 3-4 = highly disadvantaged, or deciles $1-2=$ most disadvantaged). The SES was classified by census-based Australian Bureau of Statistics Index of Relative Socioeconomic Advantage and Disadvantage (IRSAD) [32] using the participants' residential postcode. This composite index summarises information on social and economic resources of households and people living in specific postcodes. The national standardised mean is $1000( \pm 100)$, with higher scores denoting higher advantage [32]. Child factors were gender (male or 
female), duration of breastfeeding and age of introduction of complementary (solid) foods (<17-weeks, 17-25-weeks, or $\geq 26$-weeks).

\section{Statistical analyses}

All statistical analyses were performed using Stata Statistical Software version 15.0 (StataCorp, College Station, TX, USA).

\section{Participant characteristics}

The characteristics of the study sample were summarised as means and standard deviations for continuous variables, and frequency and percentages for categorical variables.

\section{Dietary trajectory analyses}

For the first objective, GBTM using a plug-in (PROC TRAJ) in Stata was used to construct the dietary trajectories. The GBTM analyses were restricted to those study participants for whom the dietary data was available from at least three interview periods. For missing dietary data, one major advantage of GBTM is that it assumes that missing data are missing at random and adjusts the model so that missing data do not contribute to the sample size or analytical outcome.

The GBTM is based on finite mixture modelling for approximating unknown trajectories across population members. The GBTM identifies clusters of individuals with similar trajectories, and the model itself forms the trajectories based on the maximum-likelihood estimation using a general quasi-Newton method [33]. The Bayesian information criteria (BIC) [34] is often used to help decide the number of groups (model selection) that best represent the heterogeneity in the trajectories of the study sample. However, the BIC does not always clearly identify a preferred number of groups. Therefore, the objective of selection of number of groups (model selection) should not be maximisation of some statistical parameter; rather, it is to summarise the data features in as parsimonious manner as possible.

For study analyses, Poisson-based model was chosen because of the continuous distribution (count data) of the food frequency data at each time point. The GBTM analysis is a two-step process: (1) select the number of groups and (2) determine the order of the polynomial defining each group's trajectory (i.e., zero-order, linear, cubic, quadratic). We fitted a series of 2- to 6-group models, testing zero-order, linear, cubic, and quadratic specifications for the trajectory shapes, until the best fitting model (which was parsimonious and analytically tractable) was established.

\section{Predictors of trajectory group membership}

For the second objective, two multinomial logistic regression analyses were performed to determine the associations between predictors (i.e., maternal and child factors) and trajectory group membership for 'core' and 'discretionary' foods groups, using Stata. Relative risk ratios (RR) were generated since the dependent variable (trajectories) was categorical with more than two groups. The trajectory representing the lowest consumption group was used as the reference category for each of the regression models. Significance level of 5\% was used for the analysis.

\section{Ethics approval and participant consent}

Ethics approval to conduct this study was given by the former Sydney South West Area Health Service - RPAH Zone (ID number X08-0115), Liverpool Hospital, University of Sydney and Western Sydney University. All participants signed a written consent form prior to study commencement.

\section{Results \\ Participants' characteristics}

A total of 1500 mothers were invited to participate in the HSHK study, of whom 1035 consented to participate (response rate - 69\%). To ensure sample representativeness, socio-demographic characteristics and chosen method of infant feeding were compared between the participating and non-participating mothers $(n=465)$. There were no significant differences between the two groups in terms of maternal age (Chi-square $\left(\mathrm{X}^{2}\right)=4.75$, $p=0.153)$, educational level $\left(\mathrm{X}^{2}=6.65, p=0.328\right)$, and method of infant feeding $\left(\mathrm{X}^{2}=2.46, p=0.813\right)$. Of the 1035, a further 67 mothers-infant dyads either opted out or were non-contactable ( 7 contact attempts made) before the baseline interview and 34 were lost to follow-up during the study period. In total, 934 participants had the dietary data (for at least three age points) required for this study (Fig. 1). Furthermore, 760 participants had complete dietary data for all five time points. There were no differences in the age, education level and method of infant feeding of mothers who completed interviews at 1 year, 2 years and 3 years, and those who withdrew from the study (data not reported). The characteristics of the mothers and their children are shown in Table 1.

\section{Dietary pattern trajectories}

Trajectories were created for 'core' foods and 'discretionary' foods consumption patterns' respectively. In terms of core foods trajectories, higher trajectories indicate a healthier diet whilst for discretionary foods trajectories, higher trajectories indicate an unhealthy diet. The individual trajectories for the seven mutually exclusive food groups (i.e., dairy, grains, fruits, vegetables, meat and its 


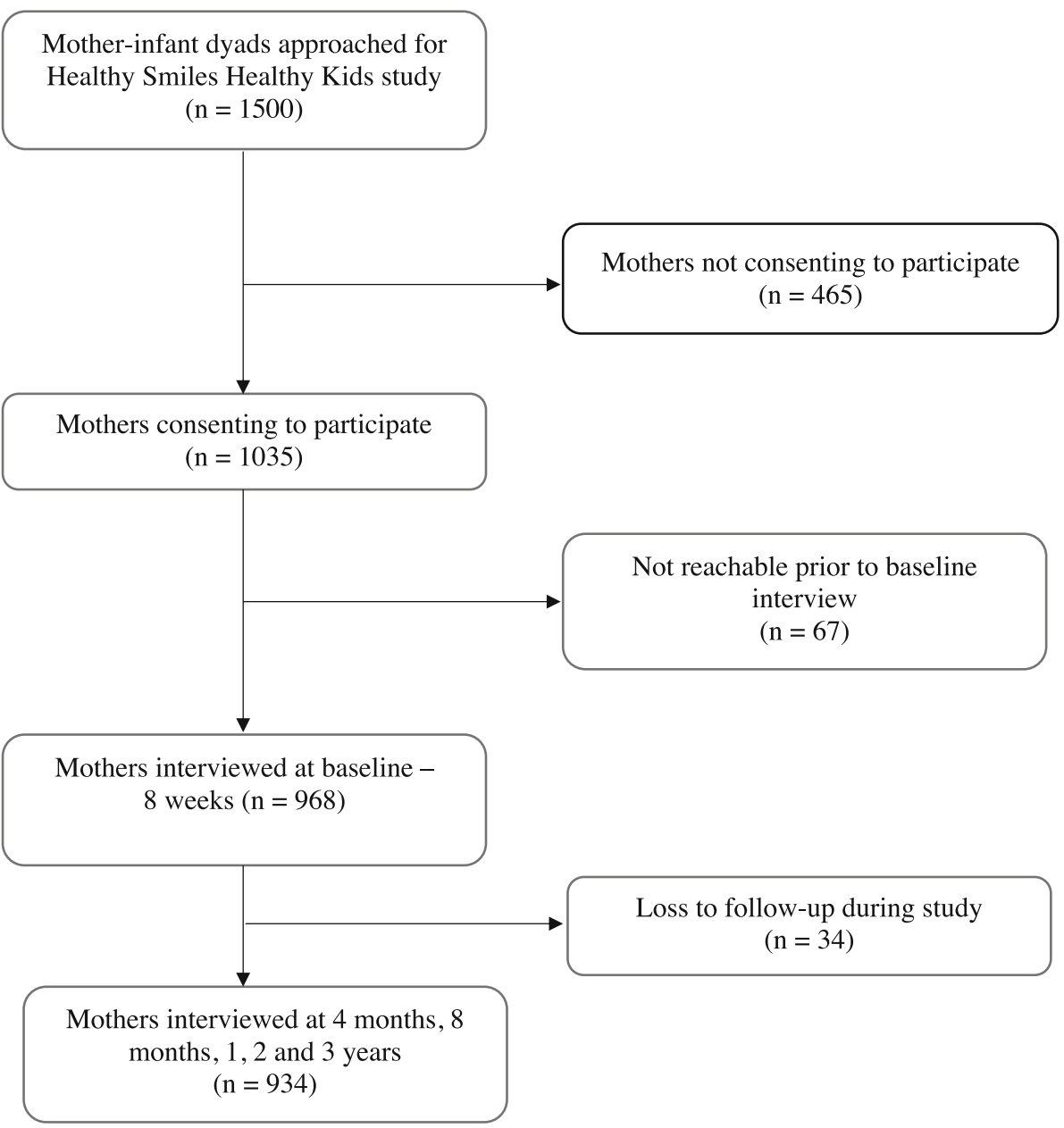

Fig. 1 Flow chart of study sample recruitment and retention

alternatives, foods with added fats and/or salt, and foods with added sugars) have been reported as Supplementary material (see Additional file 2).

\section{Trajectories of core foods and their predictors}

The GBTM identified three distinct core foods trajectories (Fig. 2): trajectory 1 (Lowest - gradual increase with late decrease) comprising of $29 \%$ of the sample; trajectory 2 (Medium - rapid increase with late decrease) comprising of 44.5\%; and trajectory 3 (Highest - rapid increase with early decrease) comprising of $26.5 \%$ of the total sample. The obtained patterns suggest that increase in children's core foods intake occurred between 4 months and 2 years of age, with frequency for all patterns decreasing between 2 and 3 years of age. From the age of 1 to 2 years, children with the highest consumption began to decrease their intake of core foods, while children in the lower consumption trajectories continued to increase their consumption until 2 to 3 years, after which a downward decline in core foods consumption was observed. Consequently, all three trajectories declined and converged with advancing age at the 3 years age point (Fig. 2). The distribution of sample characteristics by core foods trajectories are presented in Table 1.

\section{Regression analyses}

Table 2 shows the adjusted regression model of the association of maternal and child factors with core food trajectories during early childhood. After adjusting for covariates, compared with the reference trajectory 1 'Lowest consumers - Gradual increase with late decrease', children born to mothers who were born in nonEnglish speaking country were less likely to follow trajectory 2 - 'Medium consumers - Rapid increase with late decrease' (RR: 0.66, 95\%CI: 0.47-0.91; $p=0.013$ ). Compared to the reference trajectory, children were less likely to follow trajectory 3 - 'Highest consumers Rapid increase with early decrease' if they were born to a single mother (RR: 0.40, 95\%CI: $0.18-0.85 ; p=0.017$ ) and or a mother with three or more children in their household (RR: 0.46, 95\%CI: $0.27-0.77 ; p=0.003$ ), but 


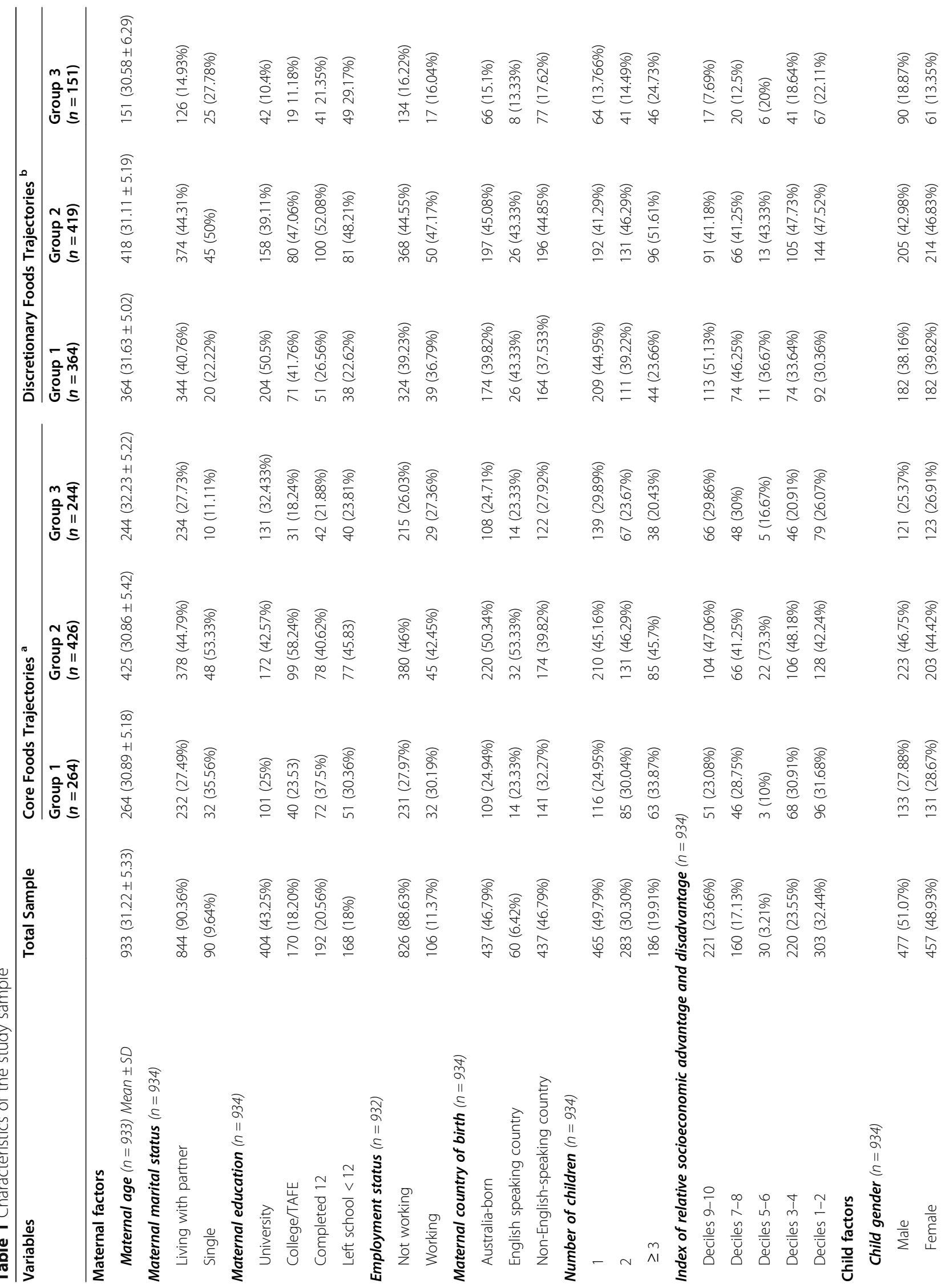




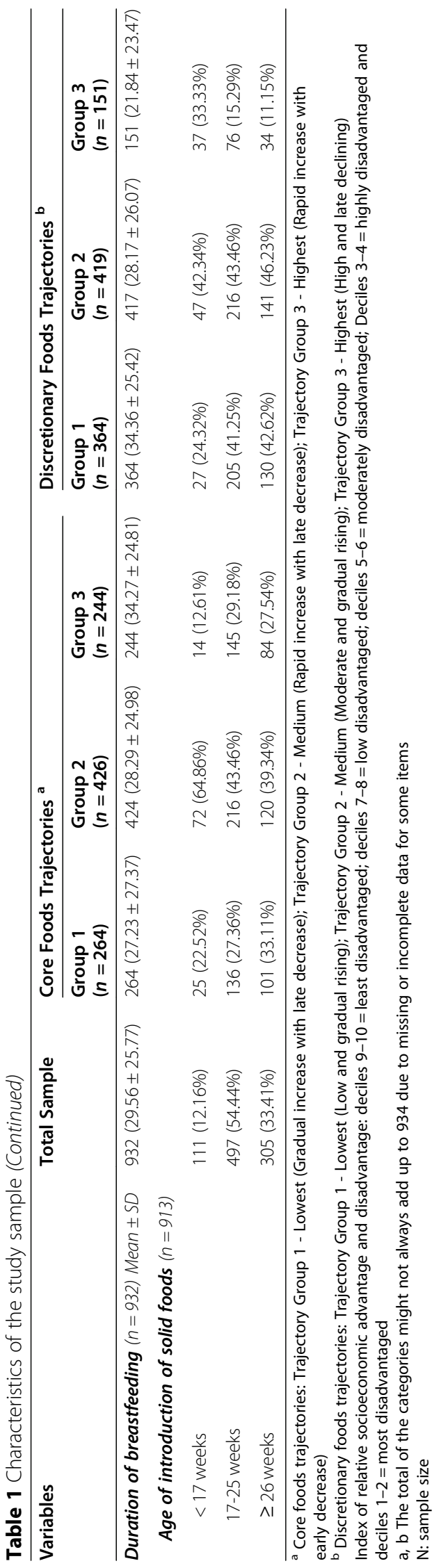




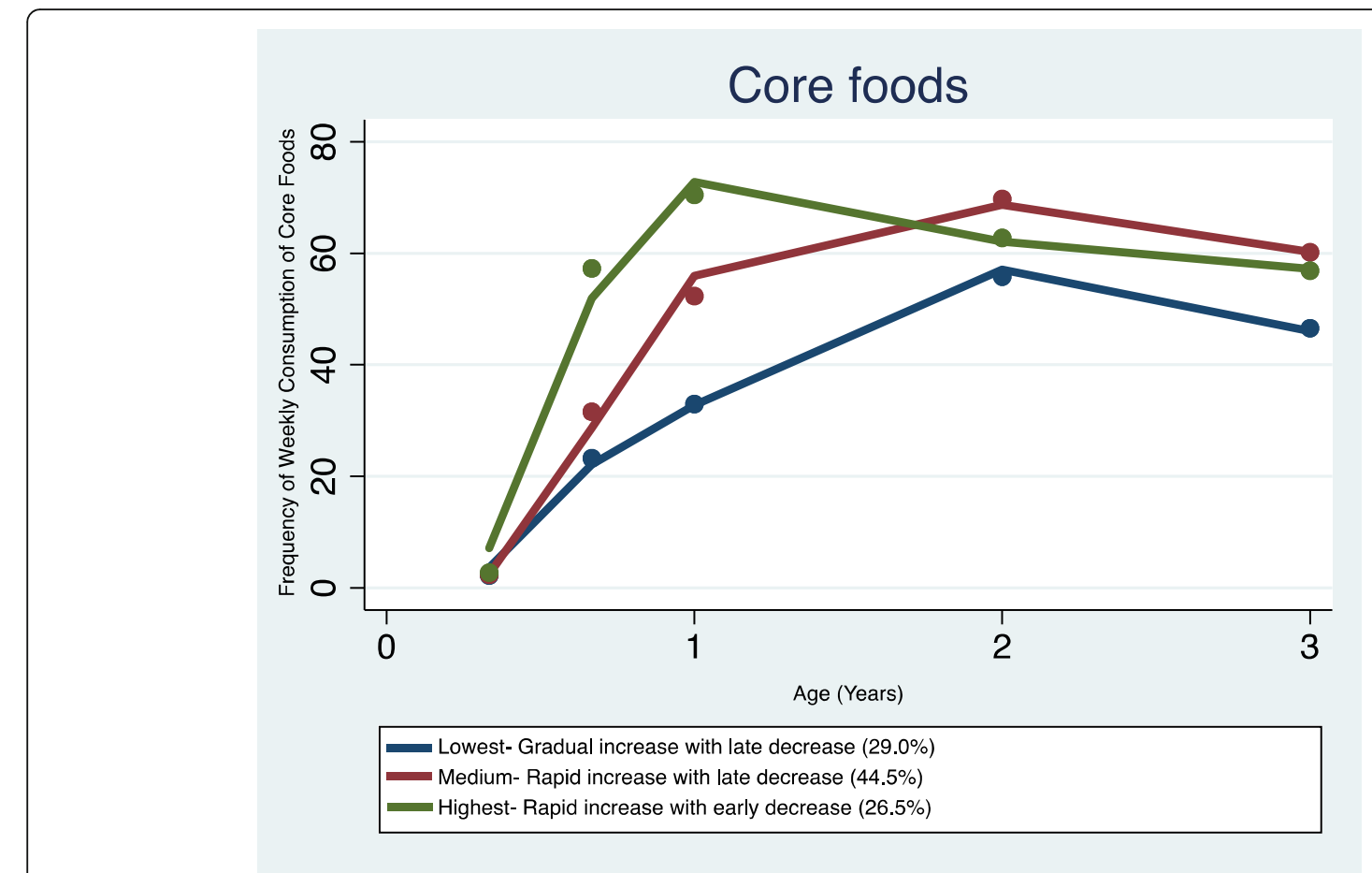

Fig. 2 Trajectories of core foods consumption in infancy and early childhood

Table 2 Factors associated with trajectories of core foods consumption in infancy and early childhood

\begin{tabular}{|c|c|c|c|c|}
\hline Core food trajectories & Adjusted RR & $95 \% \mathrm{Cl}$ & & $P$ \\
\hline Group $1^{a}$ & (reference group) & & & \\
\hline \multicolumn{5}{|l|}{ Group $2^{b, d}$} \\
\hline \multicolumn{5}{|l|}{ Maternal country of birth } \\
\hline Australia-born & 1.00 & & & \\
\hline English speaking country & 1.24 & 0.63 & 2.44 & 0.535 \\
\hline Non-English-speaking country & 0.66 & 0.47 & 0.91 & 0.013 \\
\hline \multicolumn{5}{|l|}{ Group $3^{c, e}$} \\
\hline Maternal age (in years) & 1.05 & 1.01 & 1.08 & 0.012 \\
\hline \multicolumn{5}{|l|}{ Maternal marital status } \\
\hline Married & 1.00 & & & \\
\hline Single & 0.40 & 0.18 & 0.85 & 0.017 \\
\hline \multicolumn{5}{|l|}{ Number of children } \\
\hline 1 & 1.00 & & & \\
\hline 2 & 0.66 & 0.43 & 1.00 & 0.052 \\
\hline$\geq 3$ & 0.46 & 0.27 & 0.77 & 0.003 \\
\hline Breastfeeding duration (in months) & 1.02 & 1.00 & 1.03 & 0.001 \\
\hline
\end{tabular}

RR Relative Risk Ratio, 95\% Cl 95\% Confidence Interval

${ }^{a}$ Trajectory Group 1 - Lowest (Gradual increase with late decrease)

${ }^{b}$ Trajectory Group 2 - Medium (Rapid increase with late decrease)

c Trajectory Group 3 - Highest (Rapid increase with early decrease)

${ }^{d}$ Adjusted for child gender, maternal age, maternal marital status, number of children, maternal education, maternal work status, index of relative socioeconomic advantage and disadvantage, age of introduction of solid foods, breastfeeding duration

${ }^{\mathrm{e}}$ Adjusted for child gender, maternal country of birth, maternal education, maternal work status, index of relative socioeconomic advantage and disadvantage, age of introduction of solid foods 
were more likely to follow trajectory 3 if their mother was older (RR: 1.05, 95\%CI: $1.01-1.08 ; p=0.012$ ) and breastfed longer (RR: 1.02, 95\%CI: 1.00-1.03; $p=$ $0.001)$.

Trajectories of discretionary foods and their predictors Overall, the frequency of intake of discretionary foods in the study sample was high across all time points. The GBTM identified three distinct discretionary foods trajectories (Fig. 3): trajectory 1 - 'Lowest consumers Low and gradual rising' comprising of $39.7 \%$ of the sample; trajectory 2 - 'Medium consumers - Moderate and gradual rising' comprising of $43.7 \%$; and trajectory 3 'Highest consumers - High and late declining' comprising of $16.6 \%$ of the total sample. The obtained patterns suggest that children's discretionary foods intake increased between 4 months and 2 years of age. Between the ages of 2 and 3 years, children who were initially the lowest consumers continued to have the lowest intakes, whilst children who had higher trajectories continued to maintain higher trajectories. Consequently, all the three trajectories remained distinctive with advancing age at the 3 years age point (Fig. 3). The distribution of sample characteristics by discretionary foods trajectories are also presented in Table 1.

\section{Regression analyses}

Table 3 shows the adjusted regression model of the association of maternal and child factors with discretionary foods trajectories during early childhood. After adjusting for covariates, compared with the reference trajectory 1 group - 'Lowest consumers - Low and gradual rising', children were more likely to follow trajectory 2 'Medium consumers - Medium and gradual rising' if they were born to mothers who had three or more children in household (RR: 1.97, 95\%CI: 1.26-3.11; $p=$ 0.003 ) and with low maternal education (RR: 1.81, 95\%CI: $1.10-2.99 ; p=0.019$ ), whilst longer duration of breastfeeding reduced the risk of following trajectory 2 (RR: $0.99,95 \%$ CI: $0.98-0.99 ; p=0.029$ ). Compared with the reference trajectory, being a girl (RR: $0.64,95 \% \mathrm{CI}$ : $0.42-0.97 ; p=0.037$ ), a longer duration of breastfeeding (RR: 0.99, 95\%CI: $0.97-0.99 ; p=0.029$ ), and timely introduction of complementary foods (RR: 0.30 , 95\% CI: 0.15 $0.61 ; p=0.001$ ) were associated with a lower risk of following trajectory 3 - 'Highest consumers - High and late declining'. Conversely, having three or more children in the household (RR: 2.63, 95\%CI: 1.47-4.70; $p=0.001$ ), low maternal education (RR: 3.01, 95\%CI: 1.61-5.65; $p=$ 0.001 ), and being socio-economically disadvantaged (RR: 2.69, 95\%CI: $1.31-5.55 ; p=0.007$ ) was associated with a higher risk of following trajectory 3 .

\section{Discussion}

\section{Longitudinal dietary trajectories}

In this study, consumption of discretionary foods commenced as early as 4 months of age, as reported in previous studies $[30,35,36]$. The frequency of discretionary

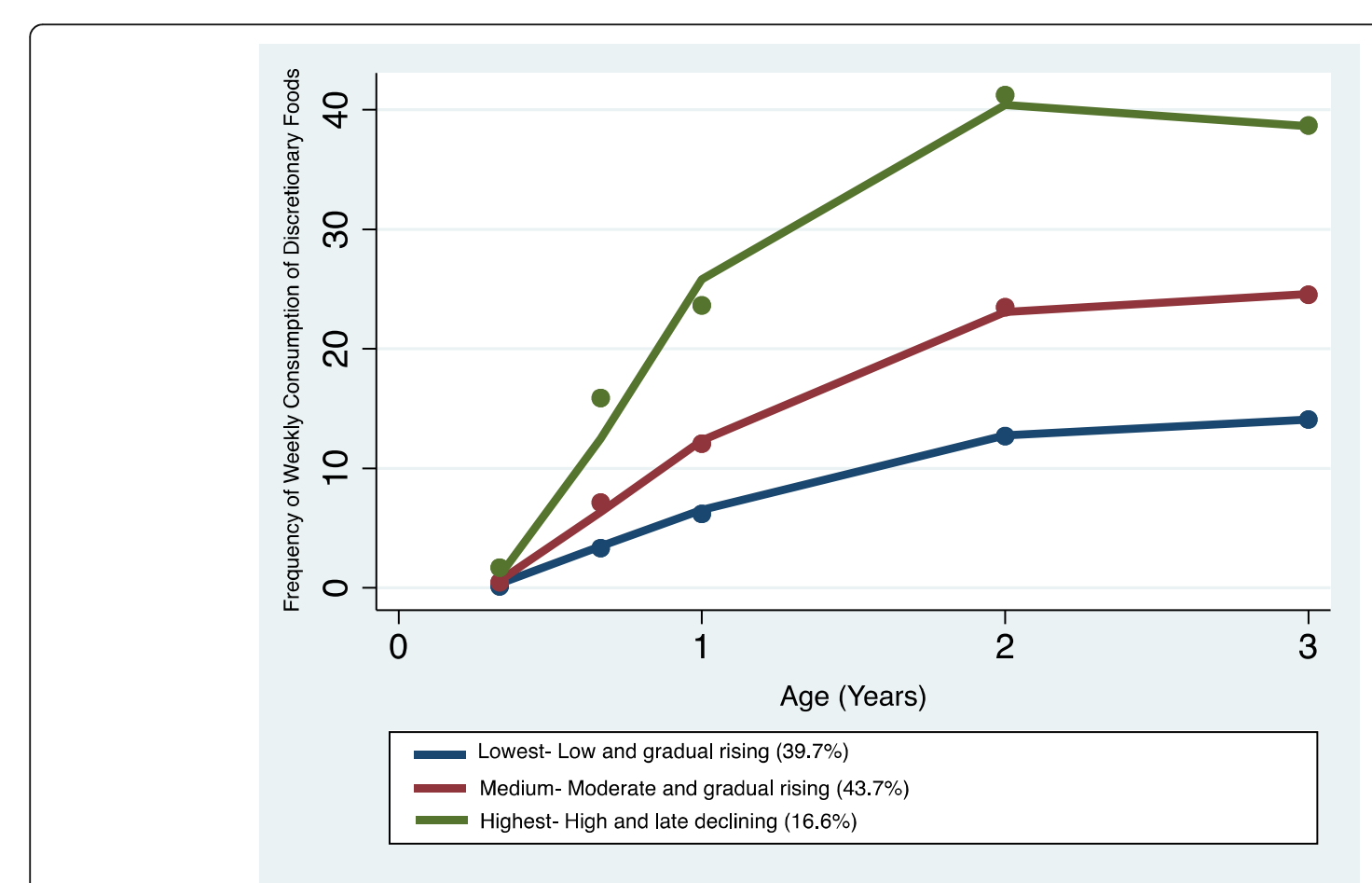

Fig. 3 Trajectories of discretionary foods consumption in infancy and early childhood 
Table 3 Factors associated with trajectories of discretionary foods consumption in infancy and early childhood

\begin{tabular}{|c|c|c|c|c|}
\hline Discretionary food trajectories & Adjusted RR & $95 \% \mathrm{Cl}$ & & $P$ \\
\hline Group $1^{a}$ & (reference group) & & & \\
\hline \multicolumn{5}{|l|}{ Group $2^{b, d}$} \\
\hline \multicolumn{5}{|l|}{ Number of children } \\
\hline 1 & 1.00 & & & \\
\hline 2 & 1.15 & 0.82 & 1.62 & 0.407 \\
\hline$\geq 3$ & 1.97 & 1.26 & 3.11 & 0.003 \\
\hline \multicolumn{5}{|l|}{ Maternal education } \\
\hline University & 1.00 & & & \\
\hline College/TAFE & 1.26 & 0.84 & 1.88 & 0.258 \\
\hline Completed 12 & 1.98 & 1.28 & 3.06 & 0.002 \\
\hline Left school $<12$ & 1.81 & 1.10 & 2.99 & 0.019 \\
\hline Breastfeeding duration (in months) & 0.99 & 0.98 & 0.99 & 0.029 \\
\hline
\end{tabular}

\section{Group $3^{\text {c,e }}$}

Child gender

$\begin{array}{ll}\text { Male } & 1.00 \\ \text { Female } & 0.64\end{array}$

Number of children

1

1.00

2

\section{Maternal education}

University

College/TAFE

Completed 12

Left school $<12$

Index of relative socioeconomic advantage and disadvantage

\author{
Deciles 9-10 \\ Deciles 7-8 \\ Deciles 5-6 \\ Deciles 3-4 \\ Deciles 1-2
}

Breastfeeding duration (in months)

Age of introduction of solid foods

$\begin{array}{ll}<17 \text { weeks } & 1.00 \\ 17-25 \text { weeks } & 0.39 \\ \geq 26 \text { weeks } & 0.30\end{array}$

RR Relative Risk Ratio, 95\% Cl 95\% Confidence Interval

Index of relative socioeconomic advantage and disadvantage: deciles 9-10=least disadvantaged; deciles 7-8=low disadvantaged; deciles 5-6=moderately disadvantaged; Deciles 3-4 = highly disadvantaged and deciles 1-2 = most disadvantaged

a Trajectory Group 1 - Lowest (Low and gradual rising)

b Trajectory Group 2 - Medium (Moderate and gradual rising)

c Trajectory Group 3 - Highest (High and late declining)

${ }^{\mathrm{d}}$ Adjusted for child gender, maternal age, maternal marital status, maternal country of birth, maternal work status, index of relative socioeconomic advantage and disadvantage, age of introduction of solid foods

e Adjusted for maternal age, maternal marital status, maternal country of birth, maternal work status 
foods consumption also continued to increase with advancing age (from 4 months to 3 years of age). Using data from the Melbourne Infant Feeding Activity and Nutrition Trial (InFANT) Program, Lioret et al. [35] observed that frequency of discretionary foods consumption amongst children doubled between 9 and 18 months of age. The LSAC study also observed consistent non-healthy dietary trajectories from the age of 2 years and onwards [16]. Similar tracking of unhealthy dietary patterns has been reported in international studies [37, 38]. Energy-dense and nutrient-poor discretionary foods contribute substantial 'empty calories' to the diets of young children [35] and may displace foods of better nutritional quality and/or value [39].

This study identified inconsistency in the dietary trajectories of healthy 'core' foods with advancing age, while unhealthy 'discretionary' foods trajectories remained relatively consistent. In contrast, previous studies have observed a consistency in both the healthy and unhealthy dietary patterns $[12,14,16]$. However, this study shows that children's core foods consumption declined after the second year of life. This might possibly reflect the age period at which children gain more independence over their dietary choices and/or influence of their parents' purchasing behaviour [13]. However, the FFQ used consisted of a relatively limited list of dietary items, and as children transitioned to the family diet, they may have eaten other core and discretionary foods that were not captured via the food list.

Frequent exposure to specific foods in early years is known to influence taste development and food preferences in later life [3]. As new food experiences in infancy influence the transition from a milk diet to a solid food diet, frequent exposure to healthy foods is likely to increase their consumption in later life [40]. Similarly, a high exposure to discretionary foods is likely to negatively influence dietary habits and food preferences in later years [41]. The study findings confirm that the period between 4 months and 2 years is a time of significant dietary transition, potentially having lifelong health implications.

\section{Predictors of infant and childhood dietary trajectories}

Children with older mothers were likely to have higher core foods trajectory scores, whilst no association was found between maternal age and high discretionary foods trajectory scores. The Avon Longitudinal Study of Parents and Children (ALSPAC) found older maternal age to be positively associated with healthy dietary patterns [37]. Prior studies have reported poor-quality diets in families with younger mothers [10, 14], possibly because they tend to cook less [42], and older mothers might have better knowledge and experience in infant nutrition [43]. Maternal marital status was negatively associated with high core foods trajectory scores. Children of single mothers consumed core foods less frequently compared to those living with both parents, as reported in a prior study [44]. As single parents are likely to have a lower household income, they may choose foods that meet the child's energy needs at a lower cost rather than expensive foods of greater variety and health rating. Single parents may experience time constraints in preparing meals and resort to the use of less nutritious convenience foods [44]. Although maternal country of birth was found to be associated with lower core foods trajectory scores, no association was found between maternal country of birth and discretionary foods trajectories. Children of non-English-speaking mothers tended to consume core foods less frequently than those with Australian-born mothers, as reported in an Australian cohort study [45]. Ethnic differences in the dietary patterns are often reported [46], which signifies the influence of culture on dietary practices.

Low level of maternal education and belonging to the most socially disadvantaged quintile were strongly associated with children following the highest discretionary foods trajectories. Previous studies have identified parental education and socio-economic position as key determinants of unhealthy dietary patterns [14, 47]. Mothers' role in children's dietary behaviours is particularly important because they usually spend more time with their child and are more closely engaged in direct feeding interactions with their child [48]. Low educated mothers may have poor food literacy, and this is reflected in their own personal dietary choices and subsequently in their child's diet [10]. The higher frequency of discretionary foods intake among children from the most socially disadvantaged quintile could be attributed to the cheaper prices of energy-dense and nutritious-poor foods [46]. Higher socio-economic status is also associated with greater food expenditure, which in turn is associated with healthier food purchasing [49].

Duration of breastfeeding and complementary feeding practices were observed to be strongly associated with dietary trajectories. As reported in earlier studies [10, 47], longer breastfeeding duration was associated with high core foods trajectories. Children who were introduced to solid foods very early (before 17 weeks of age) were most likely to have high trajectories of discretionary foods consumption. This may be explained by the effect of early feeding experiences on food and taste acceptance in later years [3]. Earlier introduction of solid foods is also associated with early introduction of discretionary foods (before 52 weeks of age) and a greater preference for discretionary foods [50]. These findings suggest that mothers who introduce solid foods early may also introduce discretionary foods early. Shorter breastfeeding duration has also been associated with a 
greater consumption of discretionary foods [50]. Longer breastfeeding duration provides ongoing exposure to a variety flavours not experienced by formula-fed infants [51], and positively influences children's vegetable intake [52]. These findings suggest that educating first-time and young mothers about the importance of breastfeeding and timely introduction of complementary foods is likely to improve their child's long-term dietary habits.

Boys were found to be higher consumers of discretionary foods compared to girls. Previous studies have also documented that boys exhibited poor dietary patterns, and consumed higher amounts of processed starches, bread, pastry, chips, fast-food and sugar-sweetened beverages $[11,14]$. As boys' energy requirements are higher than girls, their greater liking for energy-dense food groups might be an adaptive response [53]. Furthermore, boys have been reported to have a greater liking for fatty and sugary foods, whereas girls are likely to prefer fruits and vegetables [53]. Similarly, multiparity was found to be associated with lower consumption of core foods and higher consumption of discretionary foods, thus confirming the negative influence of siblings on diet quality as reported in other studies [14, 47]. Having three or more children makes it difficult for the mother to prepare adequate meals as they are busy caring for their children. Parents may also introduce discretionary foods to younger children that are typically given to older siblings, or the older siblings may share such foods with their younger siblings [54].

\section{Implications for practitioners or policymakers}

As non-healthy dietary trajectories were observed from as early as 4 months of age, it is important to target interventions in the antenatal and postpartum periods. The deterioration in the consumption of core foods from the age of 2 years might indicate that as children grow older, they develop a preference for discretionary foods; they are negatively influenced by older siblings; or they may persuade parents to purchase unhealthy foods. Children, particularly boys, breastfed for shorter duration and given solid foods very early, with two or more older siblings; born to less educated, and socioeconomically disadvantaged mothers are more likely to have high discretionary foods trajectories. Understanding of the factors that influence healthy and unhealthy dietary patterns can assist policy makers and health professionals, including general practitioners and mid-wives to design and provide more targeted and culturally appropriate support and more practical advice to the families, especially mothers (as being primary caregiver) as early as the pre-pregnancy counselling stage. Such early interventions will help to improve the dietary patterns through better compliance with the Australian infant feeding guidelines.
Additionally, the identified associations between healthy and unhealthy diet trajectories with breastfeeding duration and introduction to solids respectively can be used to inform the NSW First 2000 days Framework [55] and breastfeeding policies [56]. Furthermore, the study findings will inform policy makers on the inclusion of specific types and frequency of foods that should be eaten by children and more practical tips for parents [45] in the future Australian Dietary Guidelines planned to be released in 2024. Findings will also inform the South Western Sydney Local Health Districts' Growing Healthy Kids in SWS strategy [57] which aims to increase children's preference to core foods over discretionary foods by creating healthy food environments.

\section{Strengths and limitations}

One of the major strengths was that same instrument was used to record children's diet at evenly spaced time intervals over a three-year period. The repeated recording provides a reliable representation of longitudinal dietary exposures. The frequency of dietary intake was recorded for 7 days prior to the interview, which may better represent a child's habitual intake. This study used an innovative GBTM analysis, which enables identification of heterogeneity in the development of 'core' and 'discretionary' foods dietary patterns and avoids the use of subjective criterion and cut-offs for identification of consumer groups. Furthermore, this study explores the frequency of core and discretionary foods consumption in first 3 years of life whereas, earlier Australian studies only assessed the dietary patterns in older children or adults $[13,16]$. A large number of participants were retained $(n=934)$ and included in the trajectory analyses, providing precision and power. Finally, inclusion of a wide range of maternal and child measures assisted in identifying potential intervention strategies.

In term of limitations, children's dietary intake was parent-reported, so there may be a possibility of underreporting, social desirability bias and/or inaccurate dietary recall. However, data collection at regular time intervals minimised the chances of heaping of data and recall bias. Although the FFQ was adapted from wellestablished literature, it is unlikely to have captured the whole diet and some items were not recorded at every follow up interview. Furthermore, its validity and appropriateness for the cultural and linguistically diverse participants need to be considered. However, FFQ are easy to administer, and cost-effective therefore, commonly used in large and/or longitudinal studies [58]. Hence, it assisted in maintaining good retention in the present study. Furthermore, at each age interval, the dietary measures attempted to cover most of the essential core and discretionary foods listed in the Australian dietary guidelines however, the number of items listed were 
limited (i.e., 12 items for core foods and 20 for discretionary foods) and some items were not recorded at every follow up interview. The inclusion of extra dietary items/questions might have produced more or different dietary trajectories. Additionally, only the frequency of dietary intake was recorded rather than both frequency and serving size, so we could not capture actual intake since the serving size might vary within and between individuals with advancing age. Within core foods group, refined cereals could not be distinguished from unrefined cereals, therefore, some cereals might have been included that are high in sugar [58]. Lastly, the purpose of group-based trajectory modelling is to establish a statistical approximation of a more complex reality by creating 'trajectory' groups based on analytical convenience. However, the trajectory groups do not exist in real sense. Furthermore, the PROC TRAJ plug-in (used in this study) does not take into account the growth factor variances within the trajectory classes, which theoretically may affect trajectory group membership of some cases. However, such variation is unlikely to affect the overall outcome analysis and associated inferences [59]. Lastly, like every statistical method, GBTM has its limitations [60]. Nonetheless, GBTM seems to be a valid and effective tool to investigate the patterns (or trajectories) of dietary intake across the life-course.

\section{Conclusion}

The dietary trajectories in this study sample indicate an important risk of nutritional inadequacy for some children, with a decline in core foods intake being observed from the age of 2 years. Moreover, consumption of energy-dense, nutrient poor discretionary foods commenced as early as 4 months of age and steadily increased thereafter. These findings have important public health implications, since the behaviours influencing these dietary trajectories can be modified. The early childhood diet trajectories observed in this study are influenced by maternal socio-demographic characteristics, family size and maternal feeding practices. This study provides important evidence for promoting healthy dietary trajectories in infants, with the involvement of parents.

\footnotetext{
Abbreviations

ALSPAC: Avon Longitudinal Study of Parents and Children; BIC: Bayesian information criteria, BIC; IRSAD: Census Index of Relative Socioeconomic Advantage and Disadvantage; CFHNs: Child and Family Health Nurses; Cl: Confidence interval; FFQ: Food frequency questionnaire; GBTM: Groupbased trajectory modelling; HSHK: Healthy Smiles Healthy Kids; InFANT: Infant Feeding Activity and Nutrition Trial; LSAC: Longitudinal Study of Australian Children; PIFS: Perth Infant Feeding Studies; PCA: Principal component analysis; RRR: Relative risk ratios; SES: Socio-economic status; SSBs: Sugarsweetened beverages
}

\section{Supplementary Information}

The online version contains supplementary material available at https://doi. org/10.1186/s12937-021-00731-3.

Additional file 1: Table S1. List of dietary items $(n=32)$ recorded in the Short Food Frequency Questionnaire.

Additional file 2. Graphs depicting the individual trajectories of seven mutually exclusive food groups.

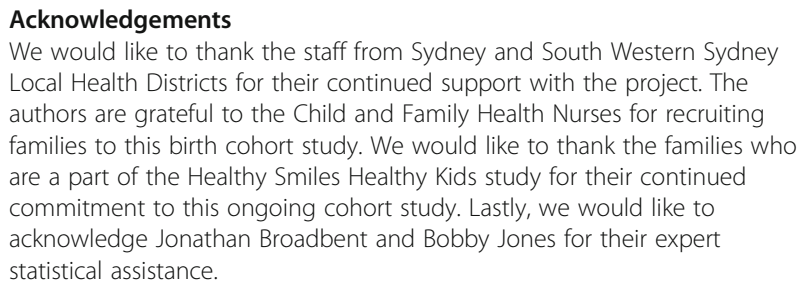

\section{Authors' contributions}

$\mathrm{NM}, \mathrm{AA}, \mathrm{LD}$, and JS designed the research. $A \mathrm{~A}, \mathrm{LD}$, and JS designed the study questionnaire. AA and SB were involved in data collection. NM analysed the data and interpreted the results with assistance from $A H, L D$, and AA. NM wrote the paper. All authors read and approved the final manuscript.

\section{Funding}

This project is supported by NHMRC Grants (1069861, 1033213, 1134075), NSW Health, Australian Dental Research Foundation, Western Sydney University, and Oral Health Foundation.

\section{Availability of data and materials}

The data of this study can't be shared publicly due to the presence of sensitive (confidential) participants' information.

\section{Declarations}

\section{Ethics approval and consent to participate}

Ethics approval to conduct this study was given by the former Sydney South West Area Health Service - RPAH Zone (ID number X08-0115), Liverpool Hospital, University of Sydney and Western Sydney University. All participants signed a written consent form prior to study commencement.

\section{Consent for publication}

All research participants consented to use their data de-identified data for publishing in scientific publications.

\section{Competing interests}

The authors declare that they have no competing interests.

\section{Author details}

${ }^{1}$ School of Health Sciences, Western Sydney University, Penrith, NSW 2751, Australia. ${ }^{2}$ Health Equity Laboratory, Campbelltown, NSW 2560, Australia. ${ }^{3}$ Australian College of Physical Education, Sydney Olympic Park, NSW 2127, Australia. ${ }^{4}$ Faculty of Health, School of Public Health, University of Technology Sydney, Ultimo, NSW 2007, Australia. ${ }^{5}$ Faculty of Health and Behavioural Sciences, School of Dentistry, University of Queensland, Brisbane, QLD 4072, Australia. ${ }^{6}$ Australian Research Centre for Population Oral Health, University of Adelaide, Adelaide, SA 5005, Australia. ${ }^{7}$ Discipline of Nutrition and Dietetics, School of Population Health, Curtin University, Perth, WA 6102, Australia. ${ }^{8}$ Oral Health Services, Sydney Local Health District and Sydney Dental Hospital, Surry Hills, NSW 2010, Australia. ${ }^{9}$ Faculty of Medicine and Health, Sydney Dental School, The University of Sydney, Surry Hills, NSW 2010, Australia. ${ }^{10}$ Translational Health Research Institute, Western Sydney University, Campbelltown, NSW 2560, Australia. ${ }^{11}$ Discipline of Child and Adolescent Health, Faculty of Medicine and Health, Sydney Medical School, Westmead, NSW 2145, Australia. 


\section{Received: 12 May 2021 Accepted: 9 August 2021} Published online: 07 September 2021

\section{References}

1. Langley-Evans S. Nutrition in early life and the programming of adult disease: a review. J Hum Nutr Diet. 2015;28:1-14. https://doi.org/10.1111/ jhn.12212.

2. Baird J, Fisher D, Lucas P, Kleijnen J, Roberts H, Law C. Being big or growing fast: systematic review of size and growth in infancy and later obesity. BMJ. 2005;331(7522):929. https://doi.org/10.1136/bmj.38586.411273.E0.

3. Birch L, Savage JS, Ventura A. Influences on the development of children's eating behaviours: from infancy to adolescence. Can J Diet Pract Res. 2007;68:s1.

4. Malik VS, Popkin BM, Bray GA, Després J-P, Willett WC, Hu FB. Sugarsweetened beverages and risk of metabolic syndrome and type 2 diabetes: a meta-analysis. Diabetes Care. 2010;33(11):2477-83. https://doi.org/10.2337/ dc10-1079.

5. Nakayama Y, Mori M. Association between nocturnal breastfeeding and snacking habits and the risk of early childhood caries in 18-to 23-month-old Japanese children. J Epidemiol. 2015;25(2):142.

6. Darnton-Hill I, Nishida C, James W. A life course approach to diet, nutrition and the prevention of chronic diseases. Public Health Nutr. 2004;7(1a):10121. https://doi.org/10.1079/PHN2003584.

7. Movassagh EZ, Baxter-Jones AD, Kontulainen S, Whiting SJ, Vatanparast H. Tracking dietary patterns over 20 years from childhood through adolescence into young adulthood: the Saskatchewan pediatric bone mineral accrual study. Nutrients. 2017;9(9):990. https://doi.org/10.3390/ nu9090990

8. Larsen JK, Hermans RC, Sleddens EF, Engels RC, Fisher JO, Kremers SP. How parental dietary behavior and food parenting practices affect children's dietary behavior. Interacting sources of influence? Appetite. 2015;89:246-57. https://doi.org/10.1016/j.appet.2015.02.012.

9. Jones BL, Nagin DS. Advances in group-based trajectory modeling and an SAS procedure for estimating them. Sociol Methods Res. 2007;35(4):542-71. https://doi.org/10.1177/0049124106292364.

10. Robinson S, Marriott L, Poole J, Crozier S, Borland S, Lawrence W, et al. Dietary patterns in infancy: the importance of maternal and family influences on feeding practice. Br J Nutr. 2007;98(5):1029-37. https://doi. org/10.1017/S0007114507750936.

11. Pryer JA, Rogers S. Dietary patterns among a national sample of British children aged 11/2-41/2; years. Public Health Nutr. 2009;12(7):957-66. https:// doi.org/10.1017/S1368980008003364.

12. Bell L, Golley R, Daniels L, Magarey A. Dietary patterns of Australian children aged 14 and 24 months, and associations with socio-demographic factors and adiposity. Eur J Clin Nutr. 2013;67(6):638-45. https://doi.org/10.1038/ ejcn.2013.23.

13. Kerr JA, Gillespie AN, Gasser CE, Mensah FK, Burgner D, Wake M. Childhood dietary trajectories and adolescent cardiovascular phenotypes: Australian community-based longitudinal study. Public Health Nutr. 2018;21(14):264253. https://doi.org/10.1017/S1368980018001398.

14. Lioret S, Betoko A, Forhan A, Charles M-A, Heude B, de Lauzon-Guillain B. Dietary patterns track from infancy to preschool age: cross-sectional and longitudinal perspectives. J Nutr. 2015;145(4):775-82. https://doi.org/10.394 5/jn.114.201988.

15. Nagin DS, Odgers CL. Group-based trajectory modeling in clinical research. Annu Rev Clin Psychol. 2010;6(1):109-38. https://doi.org/10.1146/annurev. clinpsy.121208.131413.

16. Gasser CE, Kerr JA, Mensah FK, Wake M. Stability and change in dietary scores and patterns across six waves of the longitudinal study of Australian children. Br J Nutr. 2017;117(8):1137-50. https://doi.org/10.1017/S000711451 7000897.

17. Arora A, Scott J, Bhole S, Do L, Schwarz E, Blinkhorn A. Early childhood feeding practices and dental caries in preschool children: a multi-Centre birth cohort study. BMC Public Health. 2011;11(1):28. https://doi.org/10.11 86/1471-2458-11-28.

18. Arora A, Manohar N, Hayen A, Bhole S, Eastwood J, Levy S, et al Determinants of breastfeeding initiation among mothers in Sydney, Australia: findings from a birth cohort study. Int Breastfeed J. 2017:12:39.

19. World Health Organization. Report of the expert consultation of the optimal duration of exclusive breastfeeding. Geneva: World Health Organization; 2001. 28-30 March 2001
20. National Health and Medical Research Council. Infant Feeding Guidelines. Canberra: National Health and Medical Research Council; 2012.

21. Australian Institute of Health and Welfare. 2010 Australian National Infant Feeding Survey: Indicator results PHE, Cat No. Canberra: AlHW; 2011.

22. Rankin SJ, Levy SM, Warren JJ, Gilmore JE, Broffitt B. Relative validity of an FFQ for assessing dietary fluoride intakes of infants and young children living in lowa. Public Health Nutr. 2011;14(7):1229-36. https://doi.org/10.101 7/S1368980011000474.

23. Centre for Epidemiology and Evidence. 2009-2010 Summary report from the New South Wales Child Health Survey. Sydney: NSW Ministry of Health; 2012.

24. Ha DH, Amarasena N, Crocombe LA. In: Australian Institute of Health and Welfare, editor. The dental health of Australia's children by remoteness: child dental health survey Australia 2009; 2013.

25. Scott JA, Binns CW, Graham Kl, Oddy WH. Temporal changes in the determinants of breastfeeding initiation. Birth. 2006;33(1):37-45. https://doi. org/10.1111/j.0730-7659.2006.00072.x.

26. Scott JA, Binns CW, Aroni RA. Breast-feeding in Perth: recent trends. Aust N Z J Public Health. 1996;20(2):210-1. https://doi.org/10.1111/j.1753-6405.1996. tb01820.x.

27. Arora A, Gay M, Thirukumar D. Parental choice of infant feeding behaviours in south West Sydney: a preliminary investigation. Health Educ J. 2012;71(4): 461-73. https://doi.org/10.1177/0017896912444180.

28. National Health and Medical Research Council. Eat for health - Australian Dietary Guidelines. Canberra: National Health and Medical Research Council; 2013

29. National Health and Medical Research Council. Eat for health. Educator Guide. Canberra: Commonwealth of Australia; 2013.

30. Manohar N, Hayen A, Bhole S, Arora A. Predictors of early introduction of Core and discretionary foods in Australian infants - results from HSHK birth cohort study. Nutrients. 2020;12(1):258. https://doi.org/10.3390/nu12010258.

31. Michels KB, Schulze MB. Can dietary patterns help us detect diet-disease associations? Nutr Res Rev. 2005;18(2):241-8. https://doi.org/10.1079/NRR2 005107

32. Australian Bureau of Statistics. In: Statistics Australian Bureau of Statistics, editor. Census of Population and Housing. Canberra: Socio-Economic Indexes for Areas (SEIFA); 2016.

33. Jones BL, Nagin DS, Roeder K. A SAS procedure based on mixture models for estimating developmental trajectories. Sociol Methods Res. 2001;29(3): 374-93. https://doi.org/10.1177/0049124101029003005.

34. Raftery AE. Bayesian model selection in social research. Sociol Methodol. 1995:25:111-63. https://doi.org/10.2307/271063.

35. Lioret S, McNaughton S, Spence A, Crawford D, Campbell K. Tracking of dietary intakes in early childhood: the Melbourne InFANT program. Eur J Clin Nutr. 2013;67(3):275-81. https://doi.org/10.1038/ejcn.2012.218.

36. Koh GA, Scott JA, Oddy WH, Graham KI, Binns CW. Exposure to noncore foods and beverages in the first year of life: results from a cohort study. Nutr Diet. 2010;67(3):137-42. https://doi.org/10.1111/j.1747-0080.2 010.01445.x.

37. Brazionis L, Golley RK, Mittinty MN, Smithers LG, Emmett P, Northstone K, et al. Characterization of transition diets spanning infancy and toddlerhood: a novel, multiple-time-point application of principal components analysis. Am J Clin Nutr. 2012;95(5):1200-8. https://doi.org/10.3945/ajen.111.026575.

38. Okubo H, Crozier SR, Harvey NC, Godfrey KM, Inskip HM, Cooper C, et al. Diet quality across early childhood and adiposity at 6 years: the Southampton Women's survey. Int J Obes. 2015;39(10):1456-62. https://doi. org/10.1038/ijo.2015.97.

39. Bell A, Kremer P, Magarey A, Swinburn B. Contribution of 'noncore'foods and beverages to the energy intake and weight status of Australian children. Eur J Clin Nutr. 2005;59(5):639-45. https://doi.org/10.1038/sj.ejcn.1 602091.

40. Coulthard H, Harris G, Emmett P. Long-term consequences of early fruit and vegetable feeding practices in the United Kingdom. Public Health Nutr. 2010;13(12):2044-51. https://doi.org/10.1017/S1368980010000790.

41. Savage JS, Fisher JO, Birch LL. Parental influence on eating behavior: conception to adolescence. J Law Med Ethics. 2007;35(1):22-34. https://doi. org/10.1111/j.1748-720X.2007.00111.x.

42. Betoko A, Charles M-A, Hankard R, Forhan A, Bonet M, Saurel-Cubizolles M-J, et al. Infant feeding patterns over the first year of life: influence of family characteristics. Eur J Clin Nutr. 2013;67(6):631-7. https://doi.org/10.1038/ ejcn.2012.200. 
43. Bava CM, Jaeger SR, Park J. Constraints upon food provisioning practices in 'busy' women's lives: trade-offs which demand convenience. Appetite. 2008; 50(2-3):486-98. https://doi.org/10.1016/j.appet.2007.10.005.

44. Baek YJ, Paik HY, Shim JE. Association between family structure and food group intake in children. Nutr Res Pract. 2014;8(4):463-8. https://doi.org/1 0.4162/nrp.2014.8.4.463.

45. Coxon C, Devenish G, Ha D, Do L, Scott JA. Sources and determinants of discretionary food intake in a cohort of Australian children aged 12-14 months. Int J Environ Res Public Health. 2020;17:80.

46. Wen X, Kong KL, Eiden RD, Sharma NN, Xie C. Sociodemographic differences and infant dietary patterns. Pediatrics. 2014;134(5):e1387-e98. https://doi.org/10.1542/peds.2014-1045.

47. Okubo H, Miyake Y, Sasaki S, Tanaka K, Murakami K, Hirota Y, et al. Dietary patterns in infancy and their associations with maternal socio-economic and lifestyle factors among 758 Japanese mother-child pairs: the Osaka maternal and child health study. Matern Child Nutr. 2014;10(2):213-25. https://doi.org/10.1111/j.1740-8709.2012.00403.x.

48. McHale SM, Crouter AC, McGuire SA, Updegraff KA. Congruence between mothers' and fathers' differential treatment of siblings: links with family relations and children's well-being. Child Dev. 1995;66(1):116-28. https://doi. org/10.2307/1131194.

49. Pechey R, Monsivais P. Socioeconomic inequalities in the healthiness of food choices: exploring the contributions of food expenditures. Prev Med. 2016;88:203-9. https://doi.org/10.1016/j.ypmed.2016.04.012.

50. Howard AJ, Mallan KM, Byrne R, Magarey A, Daniels LA. Toddlers' food preferences. The impact of novel food exposure, maternal preferences and food neophobia. Appetite. 2012;59(3):818-25. https://doi.org/10.1016/j.a ppet.2012.08.022.

51. Mennella J. Flavour programming during breast-feeding. In: Breast-Feeding: Early influences on later health: Springer Netherlands; 2009. p. 113-20.

52. Cooke L. The importance of exposure for healthy eating in childhood: a review. J Hum Nutr Diet. 2007;20(4):294-301. https://doi.org/10.1111/j.1365-2 77X.2007.00804.X.

53. Cooke LJ, Wardle J. Age and gender differences in children's food preferences. Br J Nutr. 2005;93(5):741-6. https:/doi.org/10.1079/BJN20051389.

54. Luque V, Escribano J, Closa-Monasterolo R, Zaragoza-Jordana M, Ferré N, Grote $V$, et al. Unhealthy dietary patterns established in infancy track to mid-childhood: the EU childhood obesity project. J Nutr. 2018;148(5):752-9. https://doi.org/10.1093/jn/nxy025.

55. NSW Department of Health. The First 2000 Days Framework. 2019.

56. New South Wales. Breastfeeding in NSW: Promotion, Protection and Support. Sydney: NSW Department of Health; 2018.

57. South Western Sydney Local Health District. Growing healthy kids in south West Sydney: SWSLHD childhood overweight and obesity prevention and management action plan 2017-2025. 2017.

58. Dunford EK, Louie JCY, Walker KZ, Gill TP. Nutritional quality of Australian breakfast cereals-are they improving? Appetite. 2012;59:464-70.

59. Broadbent J, Thomson W, Poulton R. Trajectory patterns of dental caries experience in the permanent dentition to the fourth decade of life. J Dent Res. 2008:87(1):69-72. https://doi.org/10.1177/154405910808700112.

60. Raudenbush SW. How do we study "what happens next"? Ann Am Acad Pol Soc Sci. 2005;602(1):131-44. https://doi.org/10.1177/0002716205280900.

\section{Publisher's Note}

Springer Nature remains neutral with regard to jurisdictional claims in published maps and institutional affiliations.

Ready to submit your research? Choose BMC and benefit from:

- fast, convenient online submission

- thorough peer review by experienced researchers in your field

- rapid publication on acceptance

- support for research data, including large and complex data types

- gold Open Access which fosters wider collaboration and increased citations

- maximum visibility for your research: over $100 \mathrm{M}$ website views per year

At BMC, research is always in progress.

Learn more biomedcentral.com/submissions 